\title{
Benefit Assessment of the Precision Departure Release Capability Concept
}

\author{
Kee Palopo* \\ NASA Ames Research Center, Moffett Field, CA, 94035, USA \\ Gano B. Chatterji ${ }^{\dagger}$ and Hak-Tae Lee ${ }^{\ddagger}$ \\ University of California Santa Cruz, Moffett Field, CA, 94035, USA
}

\begin{abstract}
A Precision Departure Release Capability concept is being evaluated by both the National Aeronautics and Space Administration and the Federal Aviation Administration as part of a larger goal of improving throughput, efficiency and capacity in integrated departure, arrival and surface operations. The concept is believed to have the potential of increasing flight efficiency and throughput by avoiding missing assigned slots and minimizing speed increase or path stretch to recover the slot. The main thrust of the paper is determining the impact of early and late departures from the departure runway when an aircraft has a slot assigned either at a meter fix or at the arrival airport. Results reported in the paper are for two scenarios. The first scenario considers flights out of Dallas/Fort Worth destined for Hartsfield-Jackson International Airport in Atlanta flying through the Meridian meter-fix in the Memphis Center with miles-in-trail constraints. The second scenario considers flights destined to George Bush Intercontinental/Houston Airport with specified airport arrival rate constraint. Results show that delay reduction can be achieved by allowing reasonable speed changes in scheduling. It was determined that the traffic volume between Dallas/Fort Worth and Atlanta via the Meridian fix is low and the departures times are spread enough that large departure schedule uncertainty can be tolerated. Flights can depart early or late within 90 minutes without accruing much more delay due to miles-in-trail constraint at the Meridian fix. In the Houston scenario, 808 arrivals from 174 airports were considered. Results show that delay experienced by the 16 Dallas/Fort Worth departures is higher if initial schedules of the remaining $\mathbf{7 9 2}$ flights are kept unaltered while they are rescheduled. Analysis shows that the probability of getting the initially assigned slot back after perturbation and rescheduling decreases with increasing standard deviation of the departure delay distributions. Results show that most Houston arrivals can be expected to be on time based on the assumed zero-mean Normal departure delay distributions achievable by Precision Departure Release Capability. In the current system, airport-departure delay, which is the sum of gate-departure delay and taxi-out delay, is observed at the airports. This delay acts as a bias, which can be reduced by Precision Departure Release Capability.
\end{abstract}

\section{Introduction}

$\mathrm{T}_{3}$ HE Precision Departure Release Capability (PDRC) seeks to improve the delivery of departing aircraft that must join constrained overhead flows such that they takeoff on time and arrive at the assigned slot at an en route metering point and at the destination airport. An early or late departure from the airport of origin means that the assigned slot will potentially be disrupted or missed if no compensatory maneuvers are made. Aircraft will need to reduce speed or take a longer path to compensate for early departure, and will need to speed up to make up time for late departure. Slot recovery, however, is limited by the aircraft's ability to speed up and slow down. Furthermore, controllers and airlines are reluctant to accelerate beyond filed flight plan speeds and in excess of that prescribed by the standard departure procedures. In instances when it is not possible for the aircraft to arrive at the slot, a new slot needs to be assigned by the scheduler, which can cause additional departure delays. The price of aircraft not departing on time is paid in terms of delays and more fuel being consumed.

References 1 and 2 describe the approval request process, also known as Call For Release process, in which the tower requests the traffic management coordinator at the Air Route Traffic Control Center (or Center) for departure

\footnotetext{
* Aerospace Engineer, Systems Modeling and Optimization Branch, M/S 210-15, Member.

${ }^{\dagger}$ Scientist and Task Manager, U. C. Santa Cruz, M/S 210-8, Associate Fellow.

$\ddagger$ Associate Scientist, U. C. Santa Cruz, M/S 210-8, Member.
} 
release of a flight based on a tedious manually estimated take-off/wheels-off time. The traffic management coordinator identifies a slot in the overhead stream, operating with a miles-in-trail restriction, for insertion of the requested flight and issues a departure time to comply with the miles-in-trail. Reference 1 proposes a Departure Release Communications capability to reduce the time it takes to coordinate departure release; Ref. 2 suggests the use of a Departure Release Calculator to improve the accuracy of departure release time. Two recent studies are described in Refs. 3 and 4. The former describes the Precision Departure Release Capability operational concept, system design, and system evaluation recently completed at the NASA/FAA North Texas Station facility that is preliminary to another test that will generate data for benefit analysis of the concept. Reference 4 describes the shortfalls of the current day utilization and performance of the tactical departure scheduling process in the National Airspace System and how the Precision Departure Release Capability technology, which includes an automated calculation of wheels-off time, can potentially address the shortfalls.

This paper complements Refs. 3 and 4 and shares their benefit assessment goal. The main objective of the research described in this paper is to use fast-time simulation to study the relationship between departure time uncertainty and the cost incurred in terms of increased delay, missed slots, and excess flight time. Results of the study are expected to establish departure release time accuracy needed for acceptable cost. A surface-operation management system would have to ensure that aircraft are released so that they depart from the runway within the specified time window for delay and fuel benefits to be realized, and for efficient utilization of meter-fix and arrival airport capacities.

PDRC assumes that departure release time is provided by a scheduler operating in the Center, Terminal Radar Approach Control (TRACON) or at a capacity constrained airport. For example, departure release times could be provided by the Traffic Management Advisor (TMA) for arrivals to a TMA controlled airport. One of the goals of PDRC is to assist controllers to depart aircraft on time to meet their en route or arrival constraints. The full goals of PDRC are described in Ref. 3. Any deviation from the scheduled departure time disrupts the schedule and causes additional delays. ${ }^{5}$

Benefits of PDRC are analyzed in two initial scenarios, which are the same scenarios described in Ref. 3, in terms of the impact of departure uncertainty on 1) delays, 2) missing originally allocated slots, and 3) flight time decreases or increases. Although Fort Worth center is not necessarily a target site for PDRC deployment, it was used in this study because of the availability of Dallas/Fort Worth surface operation data. It was selected as the locale for the initial PDRC field evaluation as described in Ref. 3. Other Centers that have been proposed for potential PDRC studies include Atlanta, New York, and Los Angeles due to their high ground delays during tactical departure scheduling periods.

The rest of the paper is organized as follows. Section II provides brief descriptions of the Airspace Concept Evaluation System and the first-come first-served (FCFS) scheduler, the two tools used in the study. The method for studying the effect of departure time uncertainty on increased departure delay, missing slots, and change in the flight time is detailed in Section III. This section discusses how ACES is used for simulating flights without sector and airport arrival rate constraints, and its output data are used by the FCFS scheduler to determine initial departure and arrival times based on sector and airport arrival rate constraints. The additional steps of perturbing departure times assuming departure delay distributions at the airports of origin and subsequent rescheduling for meeting the sector and airport constraints using the FCFS scheduler are also described in this section. Results of study of the two scenarios, 1) miles-in-trail constraint at Meridian meter fix for departures from Dallas/Fort Worth and 2) arrivals to Houston with airport arrival rate constraint at Houston, are presented in Section IV. Finally, the main findings are summarized in Section V.

\section{Simulation Tools and Departure Scheduler}

This section provides a summary of the two tools used for generating the results of this study. The first is the Airspace Concept Evaluation System (ACES), an air traffic simulation tool. A brief description of ACES is provided in Section II.A. The second is the FCFS scheduler described in Section II.B. Section III describes the additional delay computation procedure using these tools.

\section{A. Airspace Concept Evaluation System}

ACES is a gate-to-gate simulation of air traffic at local, regional and national levels, developed at the NASA Ames Research Center. ${ }^{6}$ ACES simulates flight trajectories using aircraft models derived from the Base of Aircraft Data $(B A D A)^{7}$ and traffic data consisting of departure times and flight-plans obtained from recorded Airline Situation Display to Industry (ASDI) files. Traffic flow management and air traffic control models in ACES use airport and 
sector capacity thresholds for simulating delaying flights on the ground and in flight. ACES can also be run without traffic flow management, which enables simulation of traffic without capacity constraints as is done in this study. Typical ACES outputs include system performance metrics of arrival, departure, en-route and total delays. Validation studies in Refs. 8 and 9 have shown that ACES generates delays and metrics comparable to those observed in the real-world.

In this study, ACES was used for simulating traffic without airport and airspace capacity constraints. The resulting output data were then used for generating inputs for the departure scheduler, which is discussed in the next section, and for verifying that the departure schedule generated by the departure scheduler satisfied the capacity constraints.

\section{B. First-Come First-Served (FCFS) Scheduler}

The departure scheduler uses the first-come first-served principle to create a departure schedule for all flights. Inputs to the scheduler are the flight-plan departure time, speed increase-decrease range, sector entry and exit times, and arrival time at the destination airport. Sector entry and exit times and arrival time at the destination airport are generated by ACES simulation of unconstrained traffic. The scheduler also needs airport arrival and departure capacities and sector capacities. For the first scenario, miles-in-trail (MIT) constraints of 10, 20, 30, and 40 nautical-miles at the Meridian fix (MEI) in Memphis Center were used to specify a circular sector with a capacity of one aircraft.

The FCFS algorithm sorts all flights according to their flight-plan departure times and then begins scheduling by allocating airport and sector resources to the flights. As flights occupy these resources for the time periods based on their transit-time, the available capacity is reduced to the point that none is available. Flights slated for later departure are delayed to find time intervals when airport and sector capacities are available. The FCFS algorithm used here is an extension of the methods described in Refs. 10 and 11. The algorithm permits speed increase and decrease considerations for slot assignment. As opposed to imposing constraints at the nodes (see: Ref. 11), links were constrained in the Meridian fix scenario.

During rescheduling, airport arrival slot assignment was performed for the Houston scenario as follows. Starting with the departure time of a flight generated using departure delay distribution, it is determined if available slots can be achieved by slowing down or speeding up. If a single slot is available, it is assigned to the flight. If the original slot (the one assigned without departure time uncertainty) is available, it is assigned. Else, the flight is assigned the next earliest slot, when several slots are available. If a slot is unavailable within the speedup-slowdown interval, the flight is held on the ground and the next earliest available slot that can be reached with the nominal speed is assigned.

\section{Approach}

The procedure for evaluating the impact of departure time uncertainty on delays, missing slots, and flight time due to rescheduling in an attempt to capture originally allocated slots is summarized in the block diagram in Fig. 1.

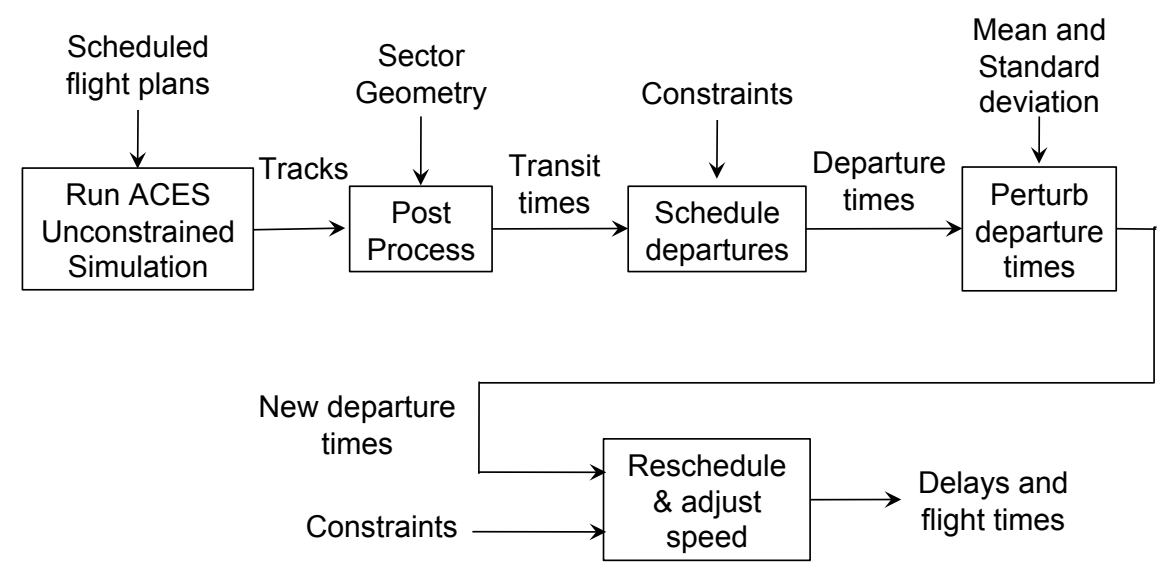

Figure 1. Experiment Procedure.

The first step consists of simulating air traffic without sector and airport capacity constraints using ACES with the input flight plans. Flight plans contain aircraft identification, aircraft type, departure airport, arrival airport, scheduled departure time, cruise speed, cruise altitude, and a sequence of waypoints marked by latitudes and longitudes along the 
route of flight. Prior to ACES simulation, flight plans are read in from a recorded ASDI data file, parsed and written out in a flight plan file.

ACES tracks output is post-processed in the second step to generate transit times consisting of sector entry and exit times and time of arrival at the destination airport. For the Meridian fix example, transit times for a flight are departure time from DFW, "MEI sector" (circular region surrounding the MEI fix) entry time and MEI sector exit time. Transit time for the IAH example consists of departure time at the airport of origin and arrival time at IAH. Sector geometry is needed for the post-processing step. An initial schedule for complying with the sector and airport capacity constraints is then created in the third step using the FCFS scheduler with the input transit times. The initial schedules are perturbed based on airport specific departure delay distributions with a specified mean from analysis of the FAA's Aviation System Performance Metrics (ASPM) database and reasonable standard deviation values up to 30 minutes in the fourth step. This results in new departure times. With scheduling priority determined by the new departure times, the FCFS method is used again to assign arrival slots and departure times in the fifth step. This assigned departure time is same as the perturbed departure time if an arrival slot is achievable by speedup or slowdown, else departure is delayed further to get a slot at a later time. Speedup or slowdown changes flight time. Delay is obtained with respect to initially scheduled departure and arrival times. Delays, missing slots, and flight times are used to measure the impact of departure delay uncertainty.

\section{A. Miles-in-Trail Constraint at MEI}

The first scenario consists of Dallas/Fort Worth International Airport departures that merge into the overhead stream through the Meridian meter-fix, subject to a miles-in-trail restriction on their way to Hartsfield-Jackson International Airport in Atlanta. Miles-in-trail is specified at Meridian for metering traffic to Atlanta. The specified miles-in-trail is used as the diameter for creating a circular region around the Meridian fix. This circular region is treated like an airspace sector and assigned a capacity of one, meaning that only one aircraft can be in the confines of the circular region at any given time. The FCFS scheduler is then used to schedule outbound traffic from Dallas/Fort Worth through the circular region such that the constraint is not violated. Departure times of the outbound flights are perturbed based on assumed departure uncertainty and the flights are rescheduled using the FCFS scheduler. Delays are computed as the difference between the rescheduled and the initially scheduled departure times.

To study this scenario, flight plans with departure times between September 24, 2010, 2:00 a.m. Central Daylight Time (CDT) and September 25, 2010 2:00 a.m. CDT, a period spanning 24 hours, were chosen. This period is representative of high-traffic-volume, low-weather-delay days in the entire National Airspace System (NAS) and was selected using the cluster analysis described in Ref. 12. Out of a total of 50,333 flights in the dataset, 1,357 flights were destined for Atlanta. Of these Atlanta arrivals, 72 cross the MEI fix at an altitude of 10,000 feet or above. Only 28 flights departing DFW arrived at Atlanta via MEI fix.

The 28 departures from DFW were initially scheduled with a MIT of 10 nautical-miles at MEI. A Normal departure delay distribution centered at 12.63 minutes, which is the average airport-departure delay with respect to scheduled DFW departures based on analysis of the ASPM database. A standard deviation of one-minute was used to generate new departure times about the initial departure schedules. Of the 12.63 minutes of airport-departure delay, $75 \%$ is gatedeparture delay and $25 \%$ is taxi-out delay. Ten thousand Monte-Carlo rescheduling runs were executed to compute delay and flight time statistics. This procedure was repeated with MIT values of 20, 30, and 40 nautical-miles and Normal departure delay distributions with standard deviation values of 2 through 30 minutes. The results are presented in Subsection A of the next section.

\section{B. Arrivals at IAH}

The second scenario consists of arrival traffic to George Bush Intercontinental/Houston Airport. Like the miles-in-trail constraint at the Meridian meter-fix, the Houston airport arrival rate constraint is specified as a function of time ranging from the sixties to a hundred arrivals per hour. The FCFS scheduler is used to schedule departures from airports of origin, including Dallas/Fort Worth, such that capacity constraints at Houston are not exceeded. Departure uncertainty represents the distribution of early and late departures from these airports. These times are used to set up first-come first-served priority for rescheduling. Assuming that early aircraft can slow down and late aircraft can speed up a bit to recover their original slot or a favorable slot, a new schedule is developed using the FCFS scheduler. Flights are then departed using this new schedule and delays are computed by subtracting the arrival times from the initially scheduled arrival times.

For this scenario, flight plans with departure times between January 2, 2011, 9:00 p.m. Central Standard Time (CST) and January 3, 2011 midnight CST, a period spanning 27 hours, were chosen. This period is representative of 
high-volume traffic days for IAH. Out of a total of 46,249 flights in the dataset, 808 flights that arrived at IAH were included in the analysis. Sixteen of the 808 flights were DFW departures.

As in the previous scenario, initial schedules were created for the 808 flights to satisfy the IAH arrival rate constraint as a function of time. Other IAH adaptation-specific metering constraints, rules, and delay-pass-back logic were not considered. Arrival rate data for the two days were obtained from the ASPM database. Next, departure times of the 16 departures from DFW were altered based on a Normal distribution centered around a departure delay of 12.63 minutes with standard deviation values of $0,1,2,5,10,15,20,25$, and 30 minutes. These 16 departures were then rescheduled using the FCFS scheduler. Delay and flight time statistics were obtained for the 10,000 Monte-Carlo runs for each standard deviation value. This entire procedure was then repeated with a zero-mean Normal distribution with standard deviation values of $0.5,1,1.5,2,3,4,5$, and 10 minutes. Speedup of one-minute and slowdown of two-minutes was allowed for slot allocation. It is easier to delay an aircraft to meet a constraint than to accelerate to meet the same constraint.

The next set of Monte-Carlo runs were made by altering the departure times of the entire set of 808 departures and using FCFS for rescheduling them to meet the arrival rate constraint at IAH. Like before, two sets of runs were made assuming a Normal departure delay distribution and a zero-mean Normal departure delay distribution. A Normal delay distribution was specified for each airport sending flights to IAH. These distributions were centered around the average airport departure delay value specific to each individual airport obtained by analysis of the ASPM data. Standard deviation values of $0,1,2,5,10,15,20,25$, and 30 minutes were used for the Normal distributions and $0.5,1,1.5,2,3,4,5$ and 10 minutes were used for the zero-mean Normal distributions. The results are discussed in Subsection B of the next section.

\section{Results}

Results of the Monte-Carlo simulations show the following. Taking speed change into consideration during scheduling lowers total delay. The speed change models the departure time window of $-2 /+1$ minutes (i.e., two minutes early/one minute late for a total of 3-minutes) described in Refs. 3 and 4. This time window modeling was applied to both scenarios. Traffic volume between Dallas/Fort Worth and Atlanta via the Meridian fix is low and departure times are spread out enough that considerable departure schedule uncertainty can be tolerated without additional delay due to miles-in-trail constraint at the Meridian fix. Delay experienced by Dallas/Fort Worth departures is higher in the Houston scenario if only their schedules are perturbed while the schedules of the other flights remain unchanged. The probability of getting an initially assigned slot back after perturbation and rescheduling decreases, or the probability of missing slots increases, with increasing standard deviation of the departure delay distributions. Finally, a majority of the Houston arrivals can be expected to be on time based on zero-mean Normal departure delay distributions expected from a capability like the Precision Departure Release Capability.

MEI metering results for the 28 departures from DFW are given in Subsection A. IAH arrival results are described in Subsection B.

\section{A. MEI Metering Scenarios for Departures from DFW to Atlanta}

Figure 2 shows the average departure delay per flight as a function of the standard deviation of the Normal departure delay distribution centered at 12.63 minutes. Recollect that the departure delay is with respect to the initially scheduled departure time. The data points in the figure correspond to MIT values of 10, 20, 30, and 40 nautical-miles. A typical MIT for MEI is 30 nautical-miles. Scheduling was done with nominal speed; speed increase or decrease were not considered. The figure shows that for MIT values of 10 and 20 nautical-miles, departure delays do not increase much. The mean of the average departure delay per flight considering all the data points and the range of standard deviation values for the MIT of 10 nautical miles is 12.65 minutes (a difference of 0.02 minutes compared to the mean of 12.63 minutes of the Normal distribution) and for the MIT of 20 nautical-miles is 12.71 minutes, an increase of 0.08 minutes compared to 12.63 minutes. Departure delays do increase with MIT of 30 and 40 nautical-miles as a function of the standard deviation of the assumed Normal distribution. Departure delay per flight for 40 nautical-miles was determined to be 13.14 minutes for the standard deviation value of 30 minutes, which is an increase of 0.51 minutes over 12.63 minutes. It should be noted that 12.63 minutes of delay acts as a bias with respect to the initially scheduled departure time. This bias can be removed by PDRC to shift the mean of the departure delay distribution to zero. Results show that if the 28 aircraft from DFW depart early or late within 90 minutes (three times the standard deviation value) with respect to their initially scheduled departure time, they would not experience much more delays due to the MIT constraint at MEI. Ninety six percent of the 280,000 departures (28 flights times 10,000 Monte-Carlo runs) incurred 
less than 0.5 minutes of additional delay. The maximum delay of 6 minutes was experienced by $0.02 \%$ of the 280,000 departures.

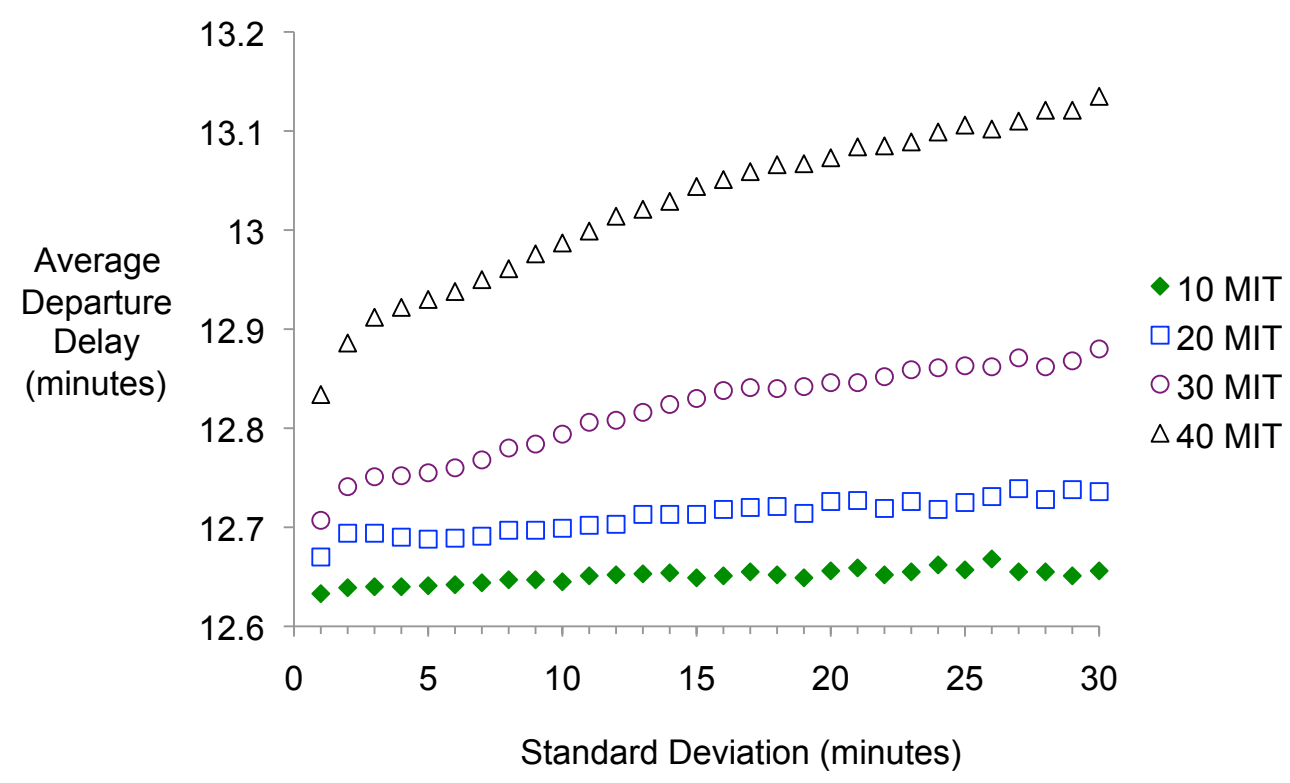

Figure 2. For 10, 20, 30, and 40 MIT at MEI. Standard deviation of 1 to 30 minutes. Perturbing 28 DFW departures only.

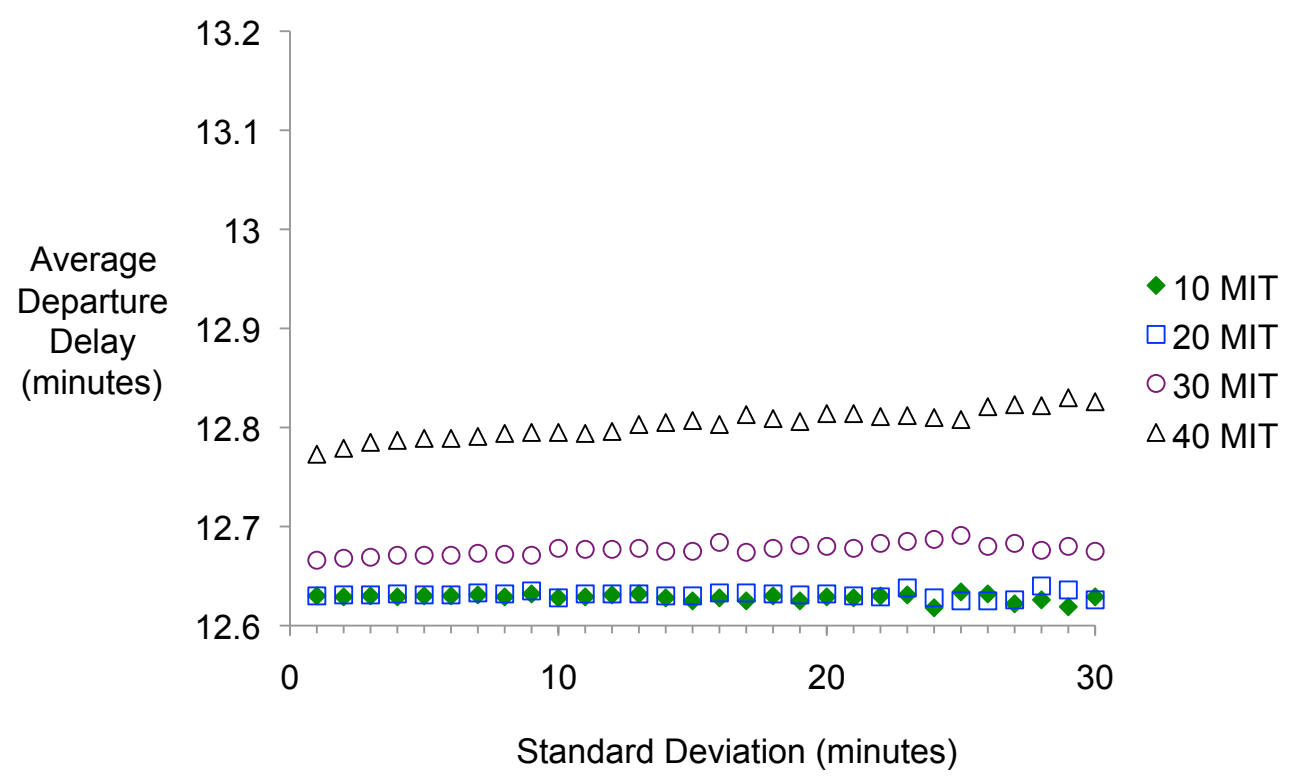

Figure 3. For 10, 20, 30, and 40 MIT at MEI. Standard deviation of 1 to 30 minutes. Speed adjustments of $5 \%$ slower to $2.5 \%$ faster than the original speed, modeling the 3-minute departure time window. Perturbing 28 DFW departures only.

Figure 3 shows the results of scheduling that allows speed increase up to $2.5 \%$ and speed decrease up to $5 \%$ with respect to the nominal speed. This speed change was applied for modeling the 3-minute departure time window. The data points shown in the figure show a similar trend as in Fig. 2 for MIT values of 10 and 20 nautical-miles. The trend of increasing average departure delay as a function of the standard deviation of the assumed Normal departure delay distribution seen earlier in Fig. 2 is not as pronounced in Fig. 3. The mean of the average departure delay per flight were found to be $12.63,12.63,12.68$, and 12.80 minutes for MIT values of 10, 20, 30, and 40 nautical-miles, respectively. These results suggest that speed changes are helpful in lowering delays. It was determined that enough slots were available for MEI metering so that when the original slot was missed, a new slot could be assigned with less than 0.3 minutes of additional delay. If the number of departures from DFW to Atlanta via the MEI fix increases in 
the future, the sensitivity of departure time uncertainty to additional departure delays needed for complying with the MIT restrictions at MEI could increase.

\section{B. IAH Arrival Scenarios}

Results obtained via Monte-Carlo runs for the IAH arrivals are described below in two subsections. The first subsection discusses the results obtained with Normal departure delay distributions centered at the average departure delay value obtained from the ASPM database for each airport sending traffic to IAH. Two situations are discussed, one in which departure times of flights from all airports of origin are perturbed and the second in which departure times of only flights out of DFW are perturbed. In the next subsection, Subsection 2, it is assumed that PDRC is available at all the airports of origin, therefore flights are expected to depart on time on an average. Zero-mean Normal departure delay distributions with specified standard deviation are therefore assumed at each airport. Results are provided for the two instances, one in which departure times of all flights are perturbed and the other in which departure times of only flights out of DFW are perturbed.

\section{Departure Times Perturbed with Airport Specific Normal Distribution}

A baseline was first established by scheduling the 808 arrivals to IAH using the FCFS scheduler. Next, the departure times of the 808 flights were altered based on assumed airport specific Normal distributions. Flights were then rescheduled to comply with the airport arrival rate constraint at IAH. Delays were computed with respect to baseline arrival times. Average arrival delays per flight obtained via Monte-Carlo runs are provided in Fig. 4. Data points marked with the triangle symbol were obtained by perturbing the departure times of all the 808 arrivals. The data points marked by the square symbol show the average arrival delay for the 16 DFW departures as a function of standard deviation values. This observation makes the point that the average arrival delay of departures from individual airports can be high while the average arrival delay considering traffic from all airports of origin can be quite low. This is due to the fact that the mean value of the Normal distribution is lower at less busy airports. The data points with the diamond symbol were obtained by perturbing only the departure times of flights out of DFW. Average arrival delays were found to be the highest in this instance. The reason is that the original arrival slots are taken up by arrivals from all airports other than those from DFW only. This means that there are fewer slots available for rescheduling DFW departures. When the departure schedules of all flights are altered, there are more opportunities for DFW flights to get earlier slots (compare the data points marked with diamonds with those marked with squares).

Figure 5 shows the probability of the originally scheduled slots to be reacquired after perturbation and rescheduling. The data points show that the probability of getting the initially assigned arrival slot back is low for small values of standard deviation. When standard deviation is low, departure times are offset by the means of the Normal distributions, which are different from the initially scheduled departure times. With larger standard deviation values, departure times of some flights are closer to the initially scheduled departure times and the spread in the departure times increases the availability of slots. The probability decreases again with larger values of standard deviation approaching 20 minutes. Large standard deviation causes the departure times to be randomized enough that new slots have to be assigned for most flights. Observe that the probability is highest when 808 flights are considered because there are many more departures from airports with Normal distributions with smaller means. The probability decreases to less than $10 \%$ when departure times of just DFW departures are perturbed. Probability of getting the initial slot is less than 5\% for DFW departures when departure times of all the 808 flights are perturbed. This is because the mean value of the Normal distribution assumed for DFW of 12.63 minutes is higher than the mean value of 9.13 minutes, obtained as the average of the mean values of the 174 airports sending traffic to IAH.

Figure 6 shows the proportions of arrivals to IAH that had shorter and longer flight times. The upper bar charts were obtained by perturbing the departure times of the 808 flights using Normal departure delay distributions specific to the airports of origin. The middle bar charts show the results for DFW departures when the departure times of the 808 flights were altered. The bottom set of bar charts show results obtained by only perturbing the departure times of flights out of DFW. Each of the subfigures show six groups of bar charts. The first two groups marked by -1 to -0.5 and -0.5 to 0 minutes are instances of reduced flight time due to speedup. The other four groups are instances of increased flight time due to slowdown. The interval -1 to -0.5 means that flights in that group arrived sooner by 30 seconds to one minute. There are nine bars in each group for standard deviation values of $0,1,2,5,10,15,20,25$, and 30 minutes. These results show that most flights sped up or slowed down within a minute range. Few flights slowed down beyond one minute. Recollect that the schedules were developed with two-minute slowdown and one-minute speedup with respect to the nominal speed. Decision makers such as the service provider and airlines can determine the economic impact of delays shown in Fig. 4 and flight time change shown in Fig. 6. 


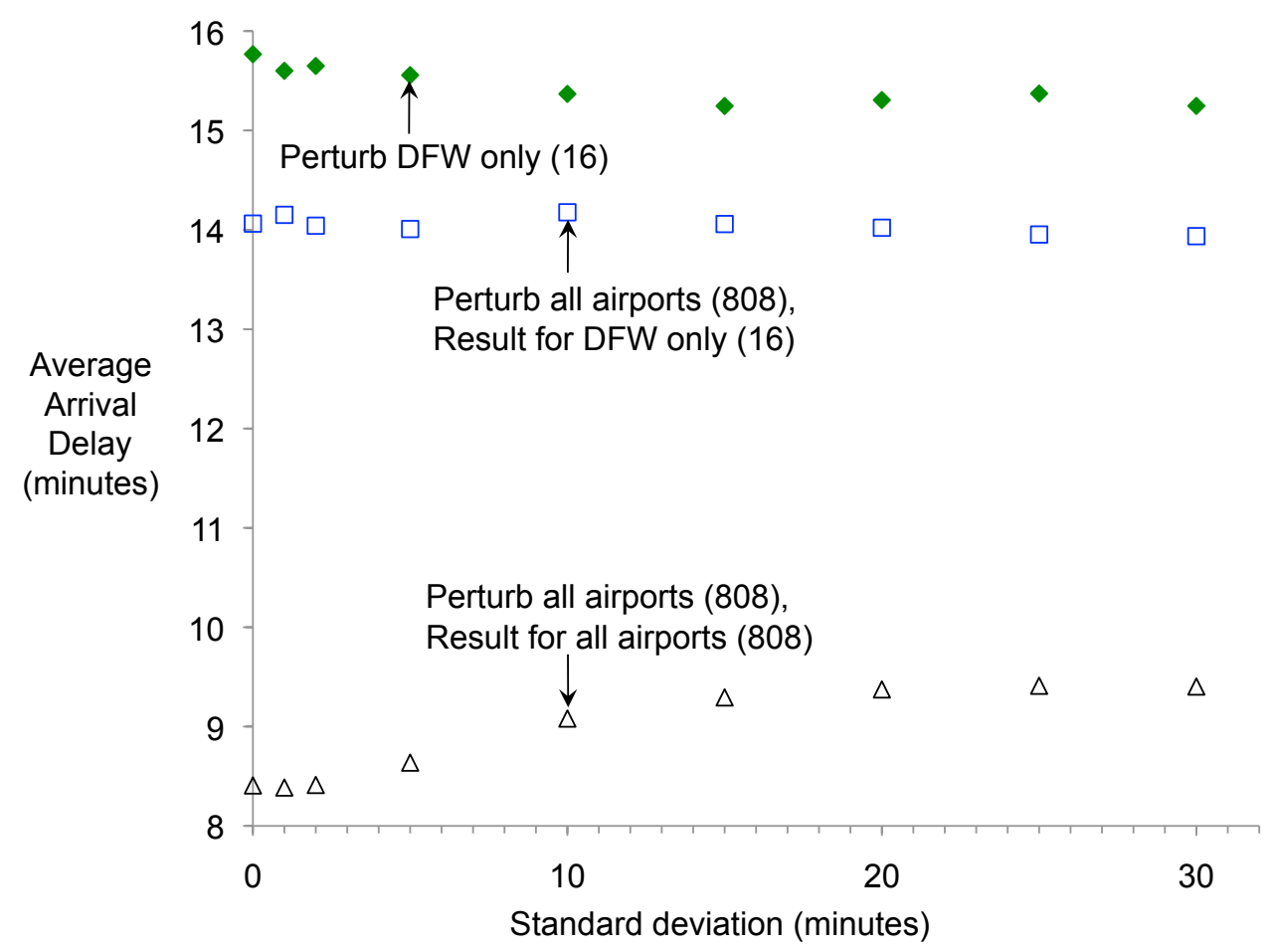

Figure 4. Perturb around each airport mean delay. Speed adjustments of 2-minutes slow-down to 1-minute speed-up, modeling the 3-minute departure time window. The number in parentheses indicates the number of flights.

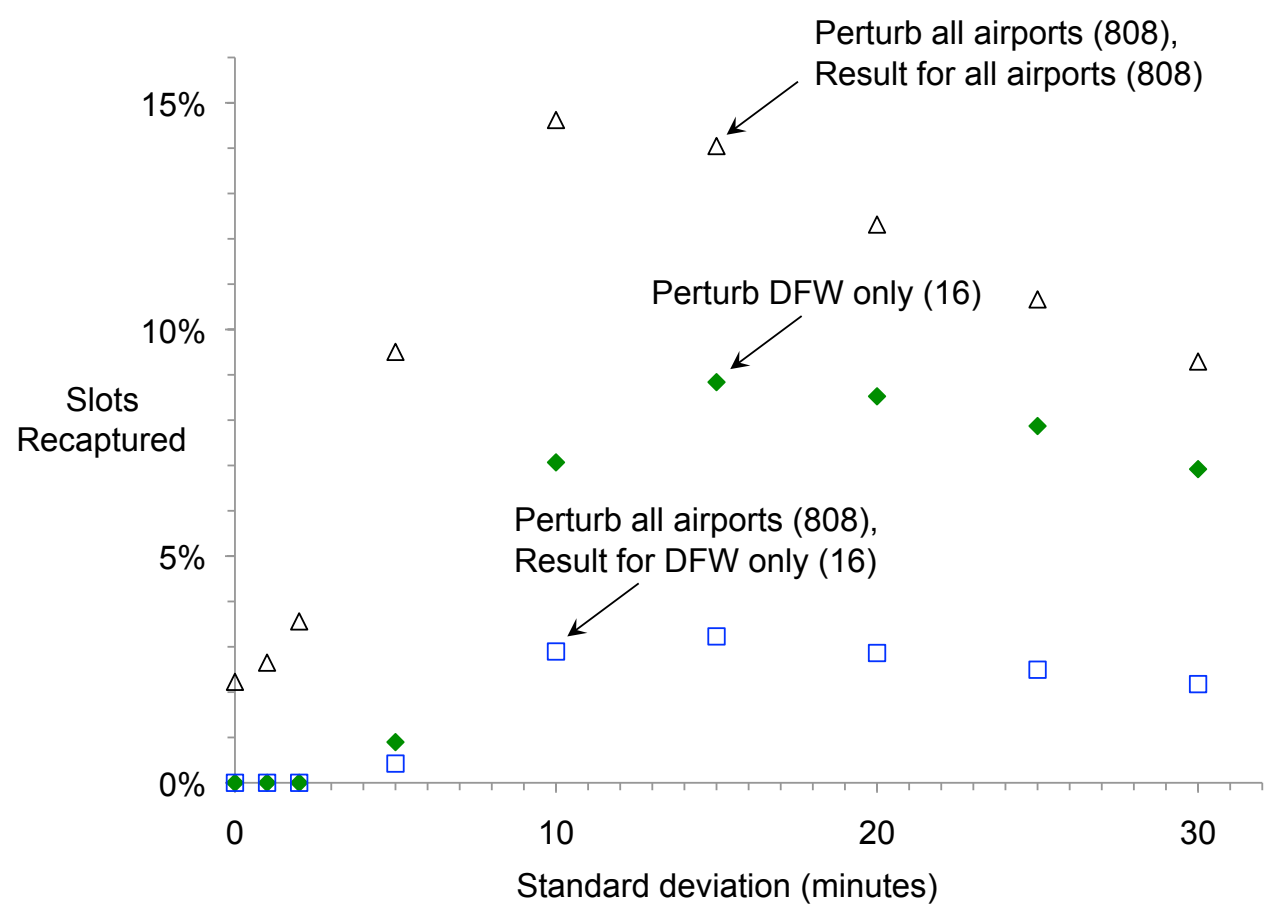

Figure 5. Perturb around each airport mean delay. Speed adjustments of 2-minutes slow-down to 1-minute speed-up, modeling the 3-minute departure time window. The number in parentheses indicates the number of flights. 


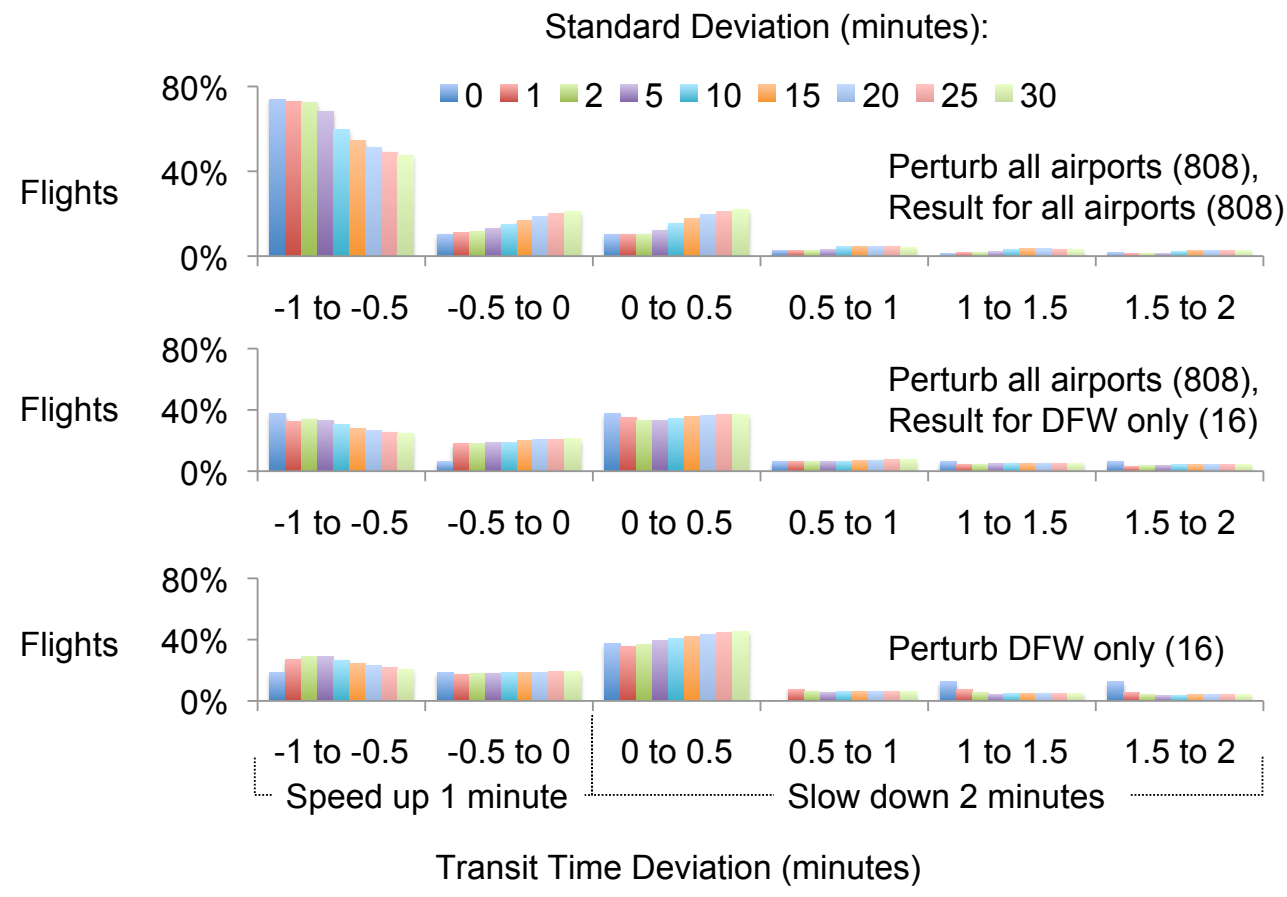

Figure 6. Perturb around each airport mean delay. Speed adjustments of 2-minutes slow-down to 1-minute speed-up, modeling the 3-minute departure time window. The number in parentheses indicates the number of flights.

\section{Departure Times Perturbed with Zero-Mean Normal Distribution}

The results described in this subsection were generated by assuming zero-mean Normal departure delay distributions at the airports of origin for IAH arrivals. The main assumption is that PDRC capability or some tool to ensure better departure time accuracy is being used at all the airports, therefore departure delay bias is removed. Thus, the mean departure delay was set to zero and the eight standard deviation values between 0.5 and 10 minutes were used.

Figures 7 shows the average arrival delays as a function of these standard deviation values. The data points marked with triangle were obtained by perturbing the departure times for the $808 \mathrm{IAH}$ arrivals. The data points marked with the square symbol denote the average arrival delay of the 16 DFW departures when the departure times of the 808 arrivals were perturbed. The data points marked with the diamond symbol correspond to arrival delay of the 16 flights when only the departures out of DFW were perturbed and FCFS scheduler was used for slot reassignment. As seen earlier in Fig. 4, average arrival delay per flight is highest when only DFW flights are perturbed and rescheduled. The significant trend in Fig. 7 compared to Fig. 4 is that arrival delay increases as a function of increasing value of standard deviation except for very high values. Very large standard deviation causes most flights to be rescheduled. This means that more slots are vacated by flights; they are available to other flights.

Figure 8 shows the probability that the initially scheduled slots get reassigned after perturbation and rescheduling. Comparing this figure to Fig. 5, it is seen that the probability of getting the initially assigned slot back decreases with increasing standard deviation value as opposed to initially increasing and subsequently decreasing. For low values of standard deviation, the probability of getting the slot back is fairly high compared to the Normal distribution results given in Fig. 5. The reason is that with the assumed zero-mean Normal delay distribution, more flights depart close to their initially scheduled departure time, therefore they are able to achieve the initially assigned slot by speeding up or slowing down.

Finally, the proportions of arrivals to IAH with shorter and longer flight times are given in Fig. 9. As in Fig. 6, the results obtained by perturbing the departure schedules of 808 IAH arrivals are given in the upper subfigure. The middle subfigure presents results for DFW departures for the scenario in which the departure times of the 808 IAH arrivals were perturbed and rescheduled. The lower subfigure contains results for the scenario in which only departures from DFW were perturbed and rescheduled. The bar charts show that the flight time difference of most flights are within -0.5 to 0.5 minutes range. In Fig. 6 it was seen that flight times of more flights were reduced (see bar charts in -1 to -0.5 minutes range). This is the effect of zero-mean Normal delay distributions compared to positive mean Normal delay distributions at the airports. 


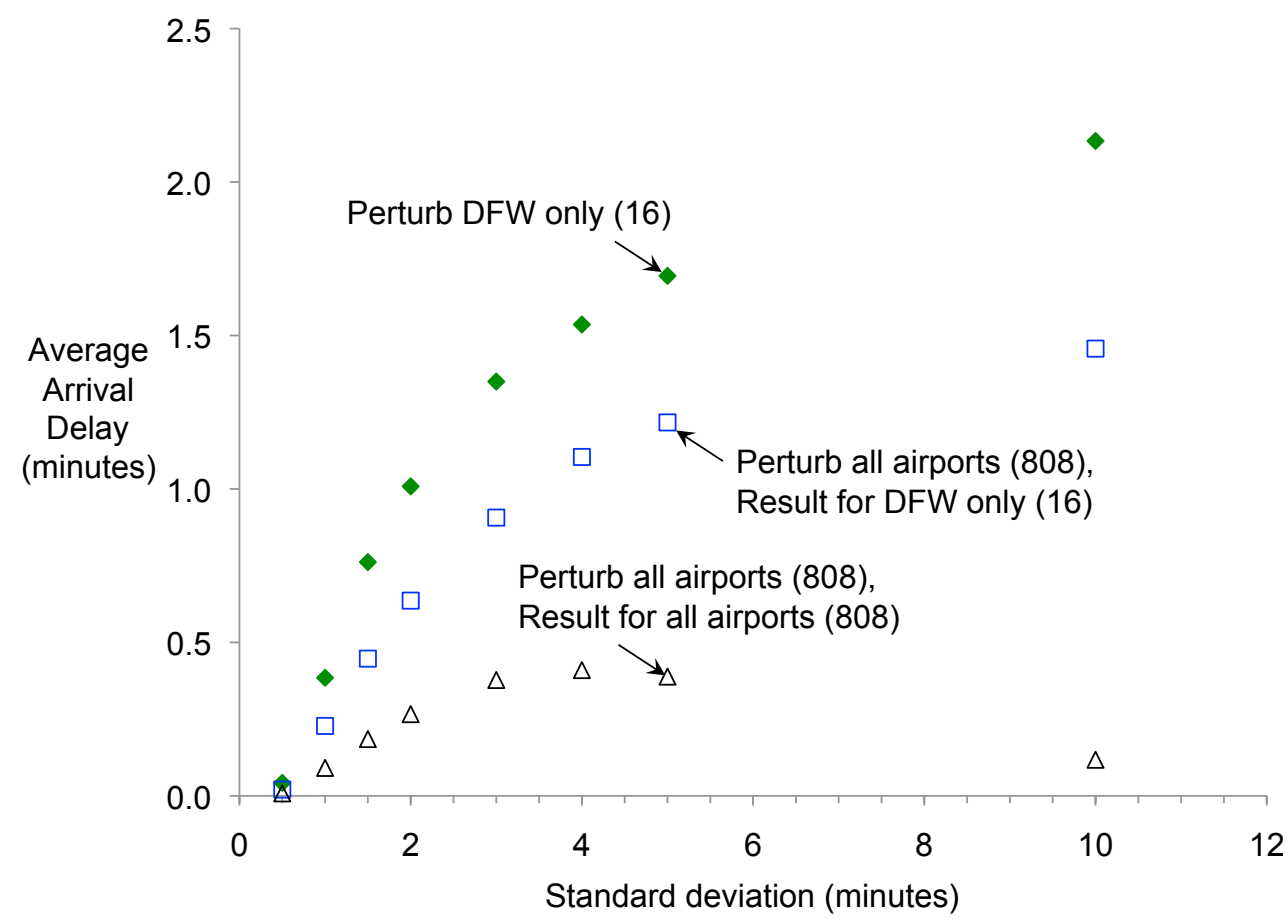

Figure 7. Perturb around zero mean delay. Speed adjustments of 2-minutes slow-down to 1-minute speed-up, modeling the 3-minute departure time window. The number in parentheses indicates the number of flights.

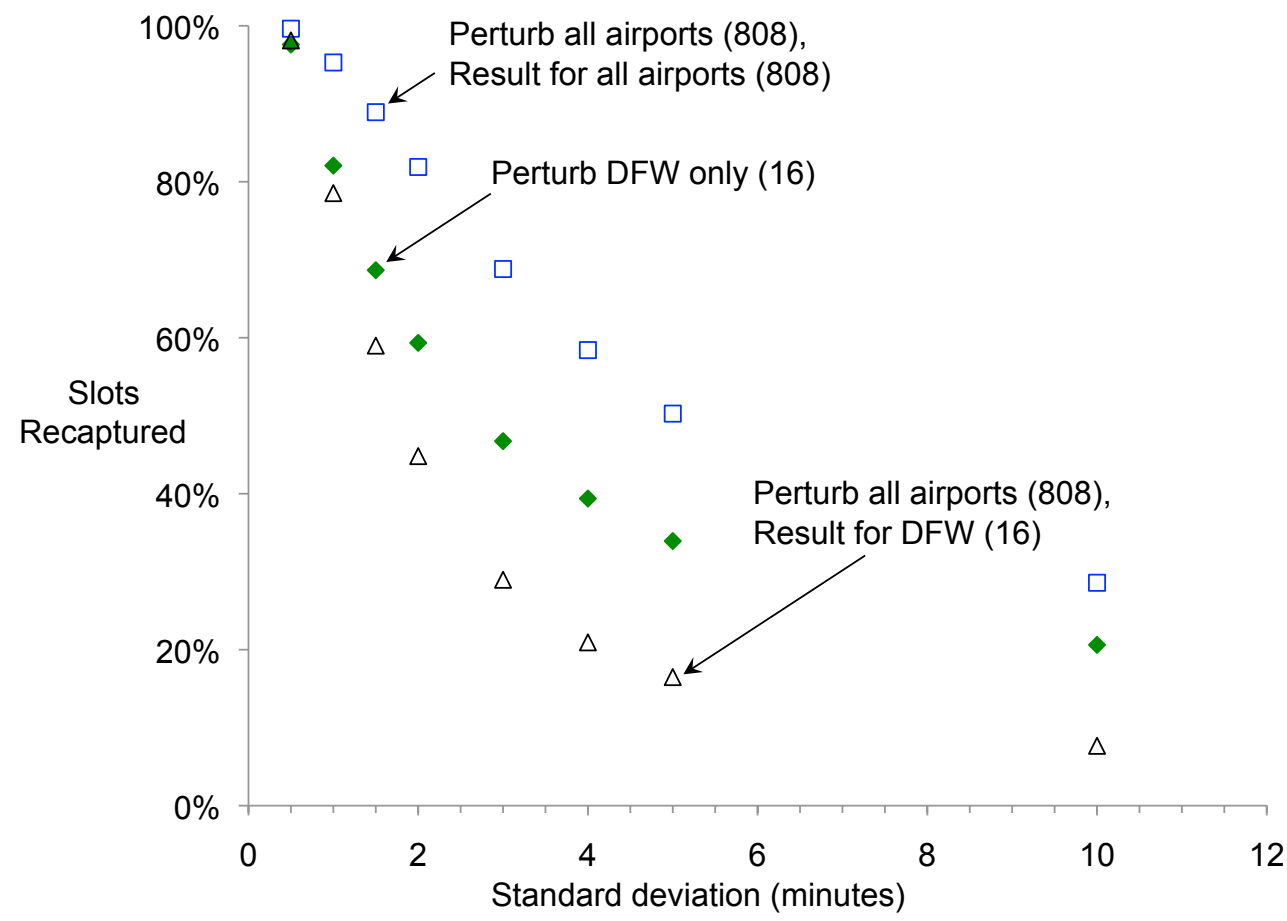

Figure 8. Perturb around zero mean delay. Speed adjustments of 2-minutes slow-down to 1-minute speed-up, modeling the 3-minute departure time window. The number in parentheses indicates the number of flights. 


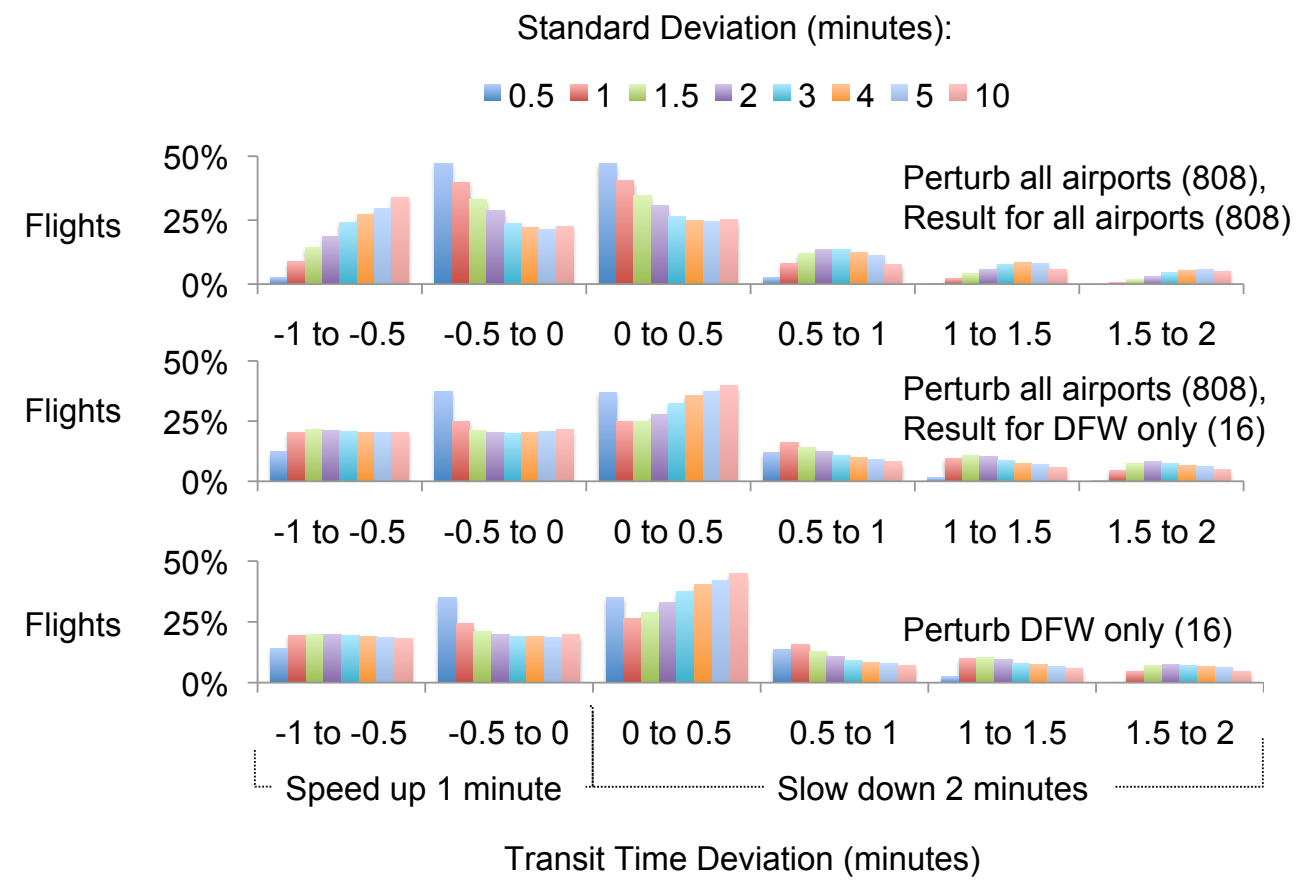

Figure 9. Perturb around zero mean delay. Speed adjustments of 2-minutes slow-down to 1-minute speed-up, modeling the 3-minute departure time window. The number in parentheses indicates the number of flights.

The data points given in Figs. 7 through 9 can be used to specify the departure delay uncertainty bounds. For example, if the slot recapture probability of $80 \%$ or higher is desired based on data in Fig. 8, the standard deviation of departure delay distributions need to be 1 minute or less, on the data points indicated by the diamond symbols.

\section{Conclusions}

A procedure for assessing the impact of departure time uncertainty when flights have an arrival slot assigned to them at an en route metering point or at the destination airport was described in this paper. The first step of the procedure consists of computing flight times using a simulation and then using them to schedule flights with a first-come first-served scheduler to comply with sector and destination airport arrival rate constraints. The next step consists of perturbing the departure times using origin airport specific Normal and zero-mean Normal departure delay distributions. Flights are then rescheduled using the same first-come first-served scheduler such that the capacity constraints are not violated. The scheduler allows a flight to speed up or slow down to achieve the initially assigned slot or a slot near it. If this is not possible, a later slot is assigned and additional delay is imposed.

Two initial scenarios were studied with this method. The first scenario is a miles-in-trail constraint at Meridian fix for Dallas/Fort Worth departures bound for Atlanta and the second scenario is flights from airports bound for Houston with an airport arrival rate constraint at Houston. To generate the results for these two scenarios, 10,000 Monte-Carlo runs were performed for each standard deviation value. For the miles-in-trail scenario, departure times of 28 departures from Dallas/Fort Worth were altered based on a Normal departure delay distribution centered at 12.63 minutes with standard deviation values from 1 to 30 minutes. Flights were rescheduled using the first-come first-served scheduler with and without speed change. Departure delays and flight time increase due to slowdown and decrease due to speedup were computed. Results show that accounting for a speed change lowers delay. The number of flights between Dallas/Fort Worth and Atlanta via the Meridian fix is low and the departures times of these flights are spread out, therefore large departure schedule uncertainty can be tolerated. Results show that if flights depart early or late within 90 minutes, they would not accrue much more delay due to miles-in-trail constraint at the Meridian fix. Ninety six percent of the 280,000 departures (28 flights times 10,000 Monte-Carlo runs) incurred less than 0.5 minutes of additional delay. The maximum delay of 6 minutes was experienced by $0.02 \%$ of the 280,000 departures.

For the Houston scenario, 808 flights arrive at Houston from 174 airports; 16 out of them are from Dallas/Fort Worth. The Houston scenario was studied in two parts. Departure times were perturbed using airport specific Normal 
distributions in the first part. The means of these departure delay distributions were obtained from the Federal Aviation Administration's Aviation System Performance Metrics database. In the second part, Precision Departure Release Capability, or highly accurate departure scheduling, was assumed at all airports sending flights to Houston; departure times were perturbed using zero-mean Normal departure delay distributions at these airports. In both these parts, initial departure and arrival schedules were determined for all the 808 flights. The departure schedules of the 16 departures from DFW were altered and rescheduled while not changing the initial schedules of the other flights in one analysis. In the other analysis, departure times of all of the 808 flights were altered and rescheduled. Results show that the average arrival delay per flight for Dallas/Fort Worth departures is higher if initial schedules of the other 792 flights are maintained while Dallas/Fort Worth departures are rescheduled. Data show that the probability of missing the initially assigned slot after perturbation and rescheduling increases with increasing standard deviation. The proportions of flights with shorter and longer flight times show that the flight time difference with respect to the nominal flight time of most Houston arrivals can be expected to be in the +/-30 seconds range based on the assumed zero-mean Normal departure delay distributions achievable by Precision Departure Release Capability.

The last result for the impact of uncertainty in departures on delays, missing slots, and transit time suggests that Precision Departure Release Capability concept, if realized, is potentially beneficial. It is expected that the capability will reduce the currently observed airport-departure delay and reduce the probability of missing slots by using more precise departure release times. Further study would be needed to evaluate the impact of this capability on transit time.

\section{Acknowledgments}

The authors thank Charlene Cayabyab for configuring and running the simulations, and for output data reduction. They thank Chunki Park for Monte Carlo scheduler implementation and simulation. They also thank Michael Downs for validating the traffic demand data. Gratitude is owed to Yun Zheng for helping with different tools and data needed for the study. Thanks are also due to Larry Meyn and Alan Capps for their thoughtful reviews and helpful suggestions for improving the paper.

\section{References}

\footnotetext{
${ }^{1}$ Kopardekar, P., Green, S., and Thompson, P., "Improving Efficiency of Departure Release Communications for En Route Overhead Traffic Flow Management," 23rd Digital Avionics Systems Conference, Salt Lake City, Utah, Oct. 24-28, 2004.

${ }^{2}$ Kopardekar, P., Green, S., Brinkman, C., Thompson, P., Evans, M., and Davis, D., "Improving Efficiency of Departure Release Communications for En Route Overhead Traffic Flow Management," 4th AIAA Aviation Technology, Integration and Operations (ATIO) Forum, Chicago, Illinois, Sep. 20-22, 2004.

${ }^{3}$ Engelland, S. and Capps, A., "Trajectory-Based Takeoff Time Predictions Applied to Tactical Departure Scheduling: Concept Description, System Design, and Initial Observations," 11th AIAA Aviation Technology, Integration and Operations (ATIO) Conference, Virginia Beach, Virginia, Sep. 20-22, 2011.

${ }^{4}$ Capps, A. and Engelland, S., "Characterization of Tactical Departure Scheduling in the National Airspace System," 11th AIAA Aviation Technology, Integration and Operations (ATIO) Conference, Virginia Beach, Virginia, Sep. 20-22, 2011.

${ }^{5}$ Thipphavong, J. and Landry, S. J., "The Effects of the Uncertainty of Departures on Multi-Center Traffic Management Advisor Scheduling," 5th AIAA Aviation Technology, Integration and Operations (ATIO) Conference, Arlington, Virginia, Sep. 26-28, 2005.

${ }^{6}$ Meyn, L., Windhorst, R., Roth, K., Drei, D. V., Kubat, G., Manikonda, V., Roney, S., Hunter, G., Huang, A., and Couluris, G., "Build 4 of the Airspace Concept Evaluation System," AIAA Modeling and Simulation Technologies Conference and Exhibit, Keystone, Colorado, 21-24 Aug. 2006.

7“User Manual for the Base of Aircraft Data (BADA) Revision 3.6," Eec note no. 10/04, Eurocontrol Experimental Centre, July 2004.

${ }^{8}$ Zelinski, S. J., "Validating the Airspace Concept Evaluation System Using Real World Data," AIAA Modeling and Simulation Technologies Conference and Exhibit, San Francisco, CA, Aug. 15-18, 2005.

${ }^{9}$ Zelinski, S. J. and Meyn, L., "Validating the Airspace Concept Evaluation System for Different Weather Days," AIAA Modeling and Simulation Technologies Conference and Exhibit, Keystone, CO, Aug. 21-24, 2006.

${ }^{10}$ Palopo, K., Chatterji, G., and Lee, H.-T., "Interaction of Airspace Partitions and Traffic Flow Management Delay," 10th AIAA Aviation Technology, Integration and Operations (ATIO) Conference, Fort Worth, Texas, Sep. 13-15, 2010.

${ }^{11}$ Meyn, L., "A Closed-Form Solution to Multi-Point Scheduling Problems," AIAA Modeling and Simulation Technologies Conference, Toronto, Ontario Canada, Aug. 2-5, 2010.

${ }^{12}$ Chatterji, G. B. and Musaffar, B., "Characterization of Days Based On Analysis of National Airspace System Performance Metrics," AIAA Guidance, Navigation and Control Conference and Exhibit, Hilton Head, South Carolina, Aug. 20-23, 2007.
} 